\title{
Exciton Absorption Spectra in Finite Parabolic Quantum Well
}

\author{
N. A. El-Meshad, H. M. Hassanain and H. H. Hassan* \\ National Research Center, Cairo, Egypt \\ *Benha Institute of Technology, Benha, Egypt
}

\begin{abstract}
We have presented a numerical calculation of the excitonic absorption spectra in finite parabolic $G a_{1-x} A l_{x} A s$ quantum well of different widths and heights. First, we evaluated the exact analytic single particle ground State solutions, in terms of Kummer functions as detailed by Wu-Pen Yuen [Phys.Rev.B 48, 17316 (1993)], for an electron and a hole. The binding energy of an exciton bound to the quantum well structure is then determined using a standard variational technique. To investigate the excitonic optical absorption near the energy gap, we used a generalized Elliot's formula originally proposed by P.Lefebvre, et.al. [Phys. Rev. B 48, 17308 (1993)]. The model is based on a simple concept that the exciton confined to a quantum well can be approximately characterized by a single parameter, namely the fractional dimensional parameter $\alpha$. Within this model, we include not only bound states contribution, but also the effects of the unbound states, which enhances the continuum absorption above the energy gap.
\end{abstract}




\section{Introduction}

Since the first fabrication of parabolic quantum wells almost a decade ago, [1] there has been a continued interest in these structures. Various experimental works concerning these structures have investigated areas such as spin dependence transport and optical properties, [2] magneto-plasma modes, [3, 4] and size effect. [5] Theoretical studies on the other hand have concentrated on a number of topics such as single particle wave functions, [6] impurities centers, $[7,8]$ electronic states, $[9,10]$ non - linear optical rectification, [11] and inter-band optical transitions. [12] The uniformity of the electron gas distribution and the intermediate dimensionality between two -dimensional (2D) and three-dimensional (3D) have made these structures a possible candidate for observing spin-density waves or Wigner crystal phase. [2-4, 10] To the best of our knowledge no previous investigation into the dimensionality characterization of the parabolic quantum wells have been done. In this work, we calculate the dimensional fraction parameter for a range of parabolic wells of width from $40 \AA$ up to $300 \AA$.

Since the formulation by Stillinger [13] of the Laplacian operator within fractional dimensional space, many authors have used this Laplacian in the kinetic part of the Hamiltonian in quantum well physics problems. [14-16] In order to calculate the dimensionality parameter $\alpha$ we followed the simple recipe described by Lefebvre et al. [17] These authors established a one to one correspondence between the dimensionality parameter $\alpha$ and the eigen energies of an exciton on a quantum well. In this recipe if we can obtain the exciton ground nergy $\mathrm{E}_{1}$ by a standard technique (such as the variational method used here) then we can write $\alpha=1+2 / \sqrt{ } E_{1}$ [17]. This relation should be viewed as merely an operational definition because $\alpha$ itself is not uniquely defined. Dimensionality as given above would be different for different potentials and it is not even the same for all eigenenergies belong to the same potential.

In order to determine the exciton binding energy, we utilized the Bastard quantum well model. [18] In this model, the trial wave function is written as a product of three wave functions. The first two are corresponding to the single particle wave function of an electron and a hole in the quantum well. The third represents a free exciton whose radius is adjusted as a variational parameter. Experiments showed that, the model worked correctly as long as the excitonic energy spectrum is coupled with the energy scales of the single electron and the single hole. For the intermediate well widths used here $(40-300 \AA)$ this criteria has been satisfied. Analytically, single particle wave function is obtained using a combination between Kummer function and the exponential functions as shown in Ref.6. 


\section{Scope on Our Calculations}

\section{A. Binding Energy}

The exciton bounded in a finite parabolic quantum well with half width $\mathrm{a}=\mathrm{L} / 2$, has a Hamiltonian $\mathrm{H}$ given by:

$$
\begin{aligned}
& \mathrm{H}=-\left(\hbar^{2} / 2 \mu \rho\right) \partial / \partial \rho(\rho \partial / \partial \rho)-\left(\hbar^{2} / 2 \mathrm{~m}_{\mathrm{e}}\right) \partial^{2} / \partial \mathrm{z}_{\mathrm{e}}-\left(\hbar^{2} / 2 \mathrm{~m}_{\mathrm{h}}\right) \\
& \partial^{2} / \partial \mathrm{z}_{\mathrm{h}}^{2}+\mathrm{V}_{\mathrm{e}}\left(\mathrm{z}_{\mathrm{e}}\right)+\mathrm{V}_{\mathrm{h}}\left(\mathrm{z}_{\mathrm{h}}\right)-\left(\mathrm{e}^{2} / 4 \pi \varepsilon_{\mathrm{o}} \varepsilon_{\mathrm{r}}\right)\left(\rho^{2}+\left(\mathrm{z}_{\mathrm{e}}-\mathrm{z}_{\mathrm{h}}\right)^{2}\right)^{-1 / 2}
\end{aligned}
$$

Here $\mu=m_{e} * m_{h} /\left(m_{e}+m_{h}\right)$ is the reduced mass of the exciton, where $\mathrm{m}_{\mathrm{e}}=0.067 \mathrm{~m}_{\mathrm{o}}, \mathrm{m}_{\mathrm{h}}=0.34 \mathrm{~m}_{\mathrm{o}}$ are the effective masses of electron and heavy hole respectively. $\mathrm{m}_{\mathrm{o}}=9.109 \times 10^{-31} \mathrm{~kg}$ is the electron rest mass. $\varepsilon_{\mathrm{r}}=13.1$ is the relative dielectric constant and $\varepsilon_{0}=8.854 \times 10^{-12} \mathrm{As} /(\mathrm{Vm})$ is the free space dielectric constant for GaAs. The first three terms in $\mathrm{H}$ represent the kinetic energy of the coordinates $\rho$ (relative plane radius), $z_{\mathrm{e}}$, and $z_{\mathrm{h}}$. The potential function $V_{e}$ and $V_{h}$ as a function of their respective coordinate $z_{e}$ and $z_{h}$ are given by

and

$$
\mathrm{V}_{\mathrm{e}}\left(\mathrm{z}_{\mathrm{e}}\right)=\begin{array}{ll}
\left\lceil\mathrm{V}_{0 \mathrm{e}} \cdot\left(\mathrm{z}_{\mathrm{e}} / \mathrm{a}\right)^{2},\right. & \left|\mathrm{z}_{\mathrm{e}}\right|<\mathrm{a} \\
\left\lfloor\mathrm{V}_{0 \mathrm{e}},\right. & \left|\mathrm{z}_{\mathrm{e}}\right|>\mathrm{a}
\end{array}
$$

$$
\mathrm{V}_{\mathrm{h}}\left(\mathrm{z}_{\mathrm{h}}\right)=\begin{array}{ll}
\mathrm{V}_{0 \mathrm{~h}} \cdot\left(\mathrm{z}_{\mathrm{h}} / \mathrm{a}\right)^{2}, & \left|\mathrm{z}_{\mathrm{h}}\right|<\mathrm{a} \\
\mathrm{V}_{0 \mathrm{~h}}, & \left|\mathrm{z}_{\mathrm{h}}\right|>\mathrm{a}
\end{array}
$$

$\mathrm{V}_{0 \mathrm{e}}$ and $\mathrm{V}_{0 \mathrm{~h}}$ are the well heights which can be determined of $\mathrm{Al}$ contents in $\mathrm{Al}_{\mathrm{x}}$ $\mathrm{Ga}_{1-\mathrm{x}}$ As, and the energy band gap. The last term in $\mathrm{H}$, shows the Coulomb potential. We chose the exciton trial envelope wave function as:

$$
\psi_{\text {exc. }}\left(z_{e}, z_{h}, \rho\right)=F_{e}\left(z_{e}\right) F_{h}\left(z_{h}\right) e^{-r / \lambda}
$$

where $r=\left[\rho^{2}+\left(z_{e}-z_{h}\right)^{2}\right]^{1 / 2}$. The variational parameter $\lambda$ is calculated by minimizing the expectation value of the exciton Hamiltonian $\langle\mathrm{H}\rangle=\left\langle\psi_{\text {exc }}|\mathrm{H}|\right.$ $\psi_{\text {exc }}>/<\psi_{\text {exc }} \mid \psi_{\text {exc }}>$. The eigen states $F_{e}$ and $F_{h}$ are the solution of Schrodinger equation for single particle with an effective mass $\mathrm{m}$, in a finite parabolic quantum well of half width a and depth $\mathrm{V}_{0}$ :

$$
\begin{array}{ll}
-\hbar^{2} / 2 \mathrm{~m}\left(\mathrm{~d}^{2} / \mathrm{dz}^{2}\right) \psi(\mathrm{z})+\mathrm{V}_{0}(\mathrm{z} / \mathrm{a})^{2} \psi(\mathrm{z})=\mathrm{E} \psi(\mathrm{z}) & |\mathrm{z}| \leq \mathrm{a} \\
-\hbar^{2} / 2 \mathrm{~m}\left(\mathrm{~d}^{2} / \mathrm{dz}^{2}\right) \psi(\mathrm{z})+\mathrm{V}_{0} \psi(\mathrm{z})=\mathrm{E} \psi(\mathrm{z}) & |\mathrm{z}|>\mathrm{a} .
\end{array}
$$

Following Ref.(6),we define a normalized parameters and $Z=Z$ /a. Equation (4) has a solution involves Kummmer function : 


$$
\begin{array}{ll}
\psi(\mathrm{Z})=\mathrm{c}_{1} \exp \left(-\mathrm{V}^{\sim} \mathrm{Z}^{2} / 2\right) \varphi\left(\mathrm{V}^{\sim} \mathrm{Z}^{2}\right), & |\mathrm{Z}| \leq 1 \\
=\mathrm{c}_{2} \exp \left[-\mathrm{W}^{\sim}(|\mathrm{Z}|-1)\right] . & |\mathrm{Z}|>1 .
\end{array}
$$

where $\varphi\left(\mathrm{V}^{\sim} \mathrm{Z}^{2}\right)$ is Kummer function $\left(\mathrm{b}, \mathrm{c}, \mathrm{V}^{\sim} \mathrm{Z}^{2}\right)$. The parameters are calculated by using the following relations ${ }^{(6)}: \mathrm{U}^{\sim 2}=2 \mathrm{ma}^{2} \mathrm{E} / \hbar^{2}, \mathrm{~V}^{2}=2 \mathrm{ma}^{2} \mathrm{~V}_{0} / \hbar^{2}, \mathrm{~b}=(1-$ $\left.\mathrm{U}^{\sim 2} / \mathrm{V}^{\sim}\right) / 4, \mathrm{c}=0.5$, and $\mathrm{W}^{\sim}=\left[\mathrm{V}^{\sim 2}-\mathrm{U}^{\sim 2}\right]^{1 / 2}$.

The continuity of the wave function and its derivative at the boundaries, give the following transcendental equation:

$$
2 \varphi^{\prime}\left(\mathrm{V}^{\sim}\right)-\left(1-\mathrm{W}^{\sim} / \mathrm{V}^{\sim}\right) \varphi\left(\mathrm{V}^{\sim}\right)=0 .
$$

Solving equation (5), for both electron and heavy hole separately, with $\mathrm{m}=$ $\mathrm{m}_{\mathrm{e}}, \mathrm{V}_{0=}=\mathrm{V}_{0 \mathrm{e}}$, and $\mathrm{m}=\mathrm{m}_{\mathrm{h}}, \mathrm{V}_{0=} \mathrm{V}_{0 \mathrm{~h}}$ respectively. The eigen values of equation (5), corresponding to $\mathrm{E}_{\mathrm{e}}$ and $\mathrm{E}_{\mathrm{h}}$.

The first three terms in $<\mathrm{H}>$ are contributed by kinetic energy components of the exciton and are given by the integral $\mathrm{I}_{1}$ :

$$
\begin{aligned}
& \mathrm{I}_{1}=\left(\hbar^{2} / 2 \mu\right) \iiint \mathrm{F}_{\mathrm{e}}^{2}\left(\mathrm{z}_{\mathrm{e}}\right) \mathrm{F}_{\mathrm{h}}^{2}\left(\mathrm{z}_{\mathrm{h}}\right)(\rho / \lambda \mathrm{r})^{2} \mathrm{e}^{-2 \mathrm{r} / \lambda} \rho \mathrm{d} \rho \mathrm{d} \mathrm{z}_{\mathrm{e}} \mathrm{d} \mathrm{z}_{\mathrm{h}} \\
& +\left(\hbar^{2} / 2 \mathrm{~m}_{\mathrm{e}}\right) \iiint \mathrm{F}_{\mathrm{h}}{ }^{2}\left(\mathrm{z}_{\mathrm{h}}\right) \mathrm{e}^{-2 \mathrm{r} / \lambda}\left[\mathrm{F}_{\mathrm{e}}{ }^{\prime}\left(\mathrm{z}_{\mathrm{e}}\right)-\left(\mathrm{z}_{\mathrm{e}}-\mathrm{z}_{\mathrm{h}}\right) \mathrm{F}_{\mathrm{e}}\left(\mathrm{z}_{\mathrm{e}}\right) / \lambda \mathrm{r}\right]^{2} \rho d \rho d \mathrm{z}_{\mathrm{e}} \mathrm{d} \mathrm{z}_{\mathrm{h}} \\
& +\left(\hbar^{2} / 2 \mathrm{~m}_{\mathrm{h}}\right) \iiint \mathrm{F}_{\mathrm{e}}{ }^{2}\left(\mathrm{z}_{\mathrm{e}}\right) \mathrm{e}^{-2 \mathrm{r} / \lambda}\left[\mathrm{F}_{\mathrm{h}}{ }^{\prime}\left(\mathrm{z}_{\mathrm{h}}\right)+\left(\mathrm{z}_{\mathrm{e}}-\mathrm{z}_{\mathrm{h}}\right) \mathrm{F}_{\mathrm{h}}\left(\mathrm{z}_{\mathrm{h}}\right) / \lambda \mathrm{r}\right]^{2} \rho d \rho d \mathrm{z}_{\mathrm{e}} \mathrm{d} \mathrm{z}_{\mathrm{h}} .
\end{aligned}
$$

The remaining three terms representing the potential energy are contained in the integral $\mathrm{I}_{2}$ :

$$
\begin{aligned}
& \mathrm{I}_{2}=\iiint \mathrm{F}_{\mathrm{e}}^{2}\left(\mathrm{z}_{\mathrm{e}}\right) \mathrm{v}_{\mathrm{e}}\left(\mathrm{z}_{\mathrm{e}}\right) \mathrm{F}_{\mathrm{h}}^{2}\left(\mathrm{z}_{\mathrm{h}}\right) \mathrm{e}^{-2 \mathrm{r} / \lambda} \rho \mathrm{d} \rho \mathrm{d} \mathrm{z}_{\mathrm{e}} \mathrm{d} \mathrm{z}_{\mathrm{h}} \\
& +\iiint \mathrm{F}_{\mathrm{e}}^{2}\left(\mathrm{z}_{\mathrm{e}}\right) \mathrm{v}_{\mathrm{h}}\left(\mathrm{z}_{\mathrm{h}}\right) \mathrm{F}_{\mathrm{h}}^{2}\left(\mathrm{z}_{\mathrm{h}}\right) \mathrm{e}^{-2 \mathrm{r} / \lambda} \rho d \rho d \mathrm{~d}_{\mathrm{e}} \mathrm{dz} \\
& -\left(\mathrm{e}^{2} / 4 \pi \varepsilon_{\mathrm{r}} \varepsilon_{\mathrm{o}}\right) \iiint \mathrm{F}_{\mathrm{e}}^{2}\left(\mathrm{z}_{\mathrm{e}}\right) \mathrm{F}_{\mathrm{h}}^{2}\left(\mathrm{z}_{\mathrm{h}}\right) \mathrm{e}^{-2 \mathrm{r} / \lambda}(1 / \mathrm{r}) \rho d \rho d \mathrm{~d}_{\mathrm{e}} \mathrm{d} \mathrm{z}_{\mathrm{h}} .
\end{aligned}
$$

all integrals have to be evaluated numerically using a Gaussian quadrature algorithm. The integrations over $z_{e}$ and $z_{h}$ are carried out from $-\infty$ to $\infty$ but integrations over $\rho$ from 0 to $\infty$. Thoroughly, the excitonic binding energy $\left(E_{b}\right)$ given by,

$$
\mathrm{E}_{\mathrm{b}}=\mathrm{E}_{\mathrm{e}}+\mathrm{E}_{\mathrm{h}}-\min <\mathrm{H}>.
$$

Having $E_{b}$, enabled us to find the value of the fractional-dimensional $\alpha$ for the parabolic well, from the equation [17],

$$
\mathrm{E}_{\mathrm{b}}=\mathrm{R} *_{\mathrm{y}} /[\mathrm{n}+(\alpha-3) / 2]^{2}
$$


Here, we used $n=1$, and the effective Rydberg $R^{*} y=\left(\mu R_{y} / m_{o} \varepsilon_{r}^{2}\right)=4.44$ mev

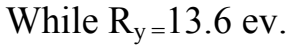

Figure (1.a and b) displays the variation of both the exciton binding energy $E_{b}$ (in the units of effective Rydberg $\mathrm{R}^{*}$ ) and the unitless fractional dimensional parameter $\alpha$ as a function of well width $\mathrm{L}$ (in $\AA$ ). As shown in both figures the binding energy $E_{b}$ increases and $\alpha$ decreases with increasing of the quantum well depth (or the $\mathrm{Al}$ content $\mathrm{x}$ in $\mathrm{Ga}_{1-\mathrm{x}} \mathrm{Al}_{\mathrm{x}} \mathrm{As}$ barrier region). But we notice that for small values of L (less than $75 \AA$ ) changing $\mathrm{x}$ from 0.15 to 0.35 does not vary $E_{b}$ and $\alpha$ much. In other words, varying $\mathrm{x}$ by 0.2 , at the narrow parabolic quantum well, causes no considerable change in the exciton binding energy. From the other hand, we see that at well width $=90$ $\AA$, the greatest descrapency between $\mathrm{E}_{\mathrm{b}}$ values at the three different values of $\mathrm{x}$ is considerable. It can be said, that the change in the binding energy is almost the same in
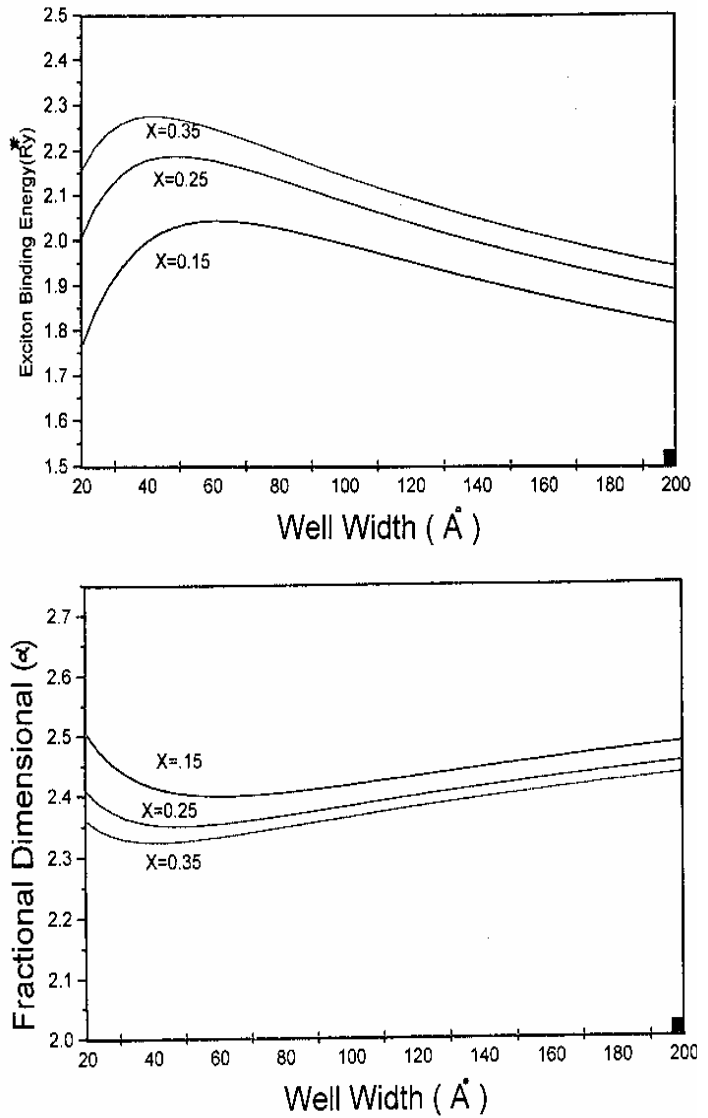

Fig. [1]: a) The exciton binding energy as a function of the well width, at three different value of the well depth, represented here by $\mathrm{x}$ (Al concentration). b) The dimensional Parameter $\alpha$ Vs the well width.

wider wells.

As a check of our analytical model, we presented Fig. (2). In this figure, we plotted the experimental [19-20] values of an exciton binding energy in a square well. At the same time, we calculated the binding energy of our exciton in a parabolic quantum well, but with the same parameters (electron, heavy hole masses and Al contents) as the square wells in reference [16]. The solid line in Fig. (2) shows our results, the filled squares represent the data given in Ref.[20], and the filled triangles indicate another experimental data of square well given in Ref. [19]. As it is clear from the figure, we notice that our results are qualitatively in a good agreement with the experimental ones. That is to say the 
exciton binding energy of an exciton in a parabolic and in square quantum wells, decreases with increasing the well size.

At different values of (Al concentration $\mathrm{x}$ ), we obtained an experimental results of heavy holes binding energy in a square quantum well. We stated our results using a parabolic quantum well with the same parameters as the square well used in measuring the experimental values from Ref. [21], in Table (1).

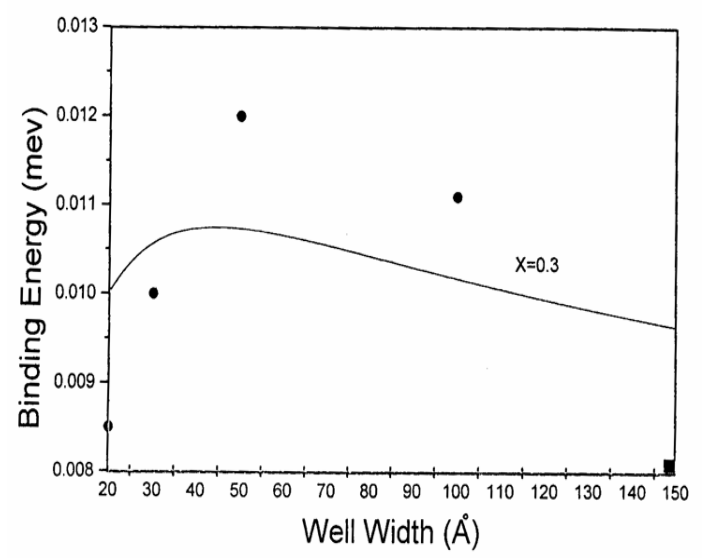

Fig. (2): Exciton binding energy variation with well width. Solid line represents our parabolic quantum well binding energy. The filled triangles and squares represent experimental values of the square well from ref.(19) and ref.(20), respectively

Table (1): Comparison between the experimental (square well)and calculated(parabolic) $E_{b}$ values.

\begin{tabular}{|c|c|c|c|}
\hline $\begin{array}{c}\text { Well Width } \\
(\AA)\end{array}$ & $\begin{array}{c}\text { Al content } \\
\mathrm{x}\end{array}$ & $\begin{array}{c}\text { Expt. } \begin{array}{c}E_{\mathrm{b}} \text { square well } \\
(\mathrm{mev})\end{array} \\
\end{array}$ & $\begin{array}{c}\mathrm{E}_{\mathrm{b}} \text { Eqn. }(8) \\
(\mathrm{mev})\end{array}$ \\
\hline 75 & 0.4 & 11.2 & 10.6 \\
\hline 82 & 1.00 & 13.0 & 13.3 \\
\hline 92 & 0.35 & 10.1 & 9.3 \\
\hline 112 & 0.35 & & 8.5 \\
\hline
\end{tabular}

Again we notice that the same behavior of decreasing the binding energy with increasing well width.

\section{B- Optical Density}

Consider the problem of a light beam incident perpendicularly to the plane of a quasi 2-D medium. This is the standard configuration for the quantum wells [22-23]. In this configuration a strong discontinuity of the electric field across the plane occurs, which cannot be described by the usual gradual exponential decay of the field. From this point of view, the "absorption coefficient" available in the literature [22-23], is rather an overall optical density of the medium, which is, in fact, the dimensionless quantity measured experimentally, as the logarithmic attenuation of light. On the other hand, if the light propagates along the plane of a quantum well or along the growth axis, a 
classical definition remains possible. The optical or spectral opacity -of the sample then involves the distance crossed by the beam. This situation is physically important, since it corre-sponds to the propagation of photons in solid-state lasers.

The capacity is calculated by accounting for the effective thickness along which the light interacts with the electronic states of the system. This thickness, $\mathrm{L}_{c}$, in case of finite well potentials, is a little bit smaller than the well width.

The general formula, for the excitonic may be considered $\alpha$ dimensional, is giving by generalizing Elliott's formula [24, 17]:

$$
\begin{aligned}
& \mathrm{O}(\hbar \omega)=\mathrm{O}_{\mathrm{o}}\left\{\sum_{\mathrm{n}=1}^{\infty}\left[\mathrm{R}_{\mathrm{y}}^{*} \Gamma(\mathrm{n}+\alpha-2) /(\mathrm{n}-1) !(\mathrm{n}+(\alpha-3) / 2)^{\alpha+1}\right] \delta\left(\hbar \omega-\mathrm{E}_{\mathrm{n}}\right)\right. \\
& \left.+|\Gamma[(\alpha-1) / 2+\mathrm{i} \gamma]|^{2} \mathrm{e}^{\pi \gamma} \gamma^{2-\alpha} \mathrm{Y}(\hbar \omega) / 2^{\alpha} \pi^{2-\alpha / 2} \Gamma(\alpha / 2)\right\},
\end{aligned}
$$

where $\mathrm{Y}(\hbar \omega)$ is the Heaviside step function,$\gamma=\left(\mathrm{R}^{*} \mathrm{y} / \mathrm{E}\right)^{1 / 2}$ and $\Gamma(\mathrm{x})$ is a gamma function.

$$
\begin{aligned}
& \mathrm{O}_{\mathrm{o}}=\left\{2^{2 \alpha-1} \omega\left|\mathrm{d}_{\mathrm{cv}}\right|^{2}(\Gamma(\alpha / 2))^{2} \Gamma((\alpha-1) / 2) / \pi^{(\alpha-3 / 2)} \mathrm{n}_{\mathrm{b}} \mathrm{cR}_{\mathrm{y}}^{*}\right\}^{*} \\
& \left\{\mathrm{a}_{\mathrm{o}}^{\alpha} \mathrm{L}^{2-\alpha}{ }_{\mathrm{c}}(\Gamma(\alpha-1))^{3}\right\},
\end{aligned}
$$

$\mathrm{n}_{\mathrm{b}}$ is the refractive index in GaAs $\left(\mathrm{n}_{\mathrm{b}}=3.2\right), \mathrm{c}$ is the speed of light, the Bohr radius $\mathrm{a}_{\mathrm{o}}=\left(\varepsilon_{\mathrm{r}} \mathrm{m}_{\mathrm{o}} \mathrm{a}_{\mathrm{b}} / \mu\right)=123.75 \AA$, and $\mathrm{a}_{\mathrm{b}}=0.529 \AA\left|\mathrm{d}_{\mathrm{cv}}\right|^{2}$ is the square matrix element of the electric dipole moment at critical point, where $\left|\mathrm{d}_{\mathrm{cv}}\right|=\mathrm{e} \int \psi^{*}{ }_{\text {exc }} \mid \mathrm{z}_{\mathrm{e}}-$ $z_{\mathrm{h}} \mid \psi_{\text {exc }} \mathrm{d} \psi_{\text {exc }}$. The last two equations [17] are more general than expression due to $\mathrm{He}$ [25] expression (replaced the absorption of the continued exciton states by the - dimensional interband absorption coefficient).

To account for the finite lifetime of exciton, Eqn. (10) must be multiplied by some broadening function. We used Lorentzian shape, with half width at half maximum $B=0.5$. The measurable optical density is given by:

$$
\mathrm{O}^{\text {tot }}(\hbar \omega)=(2 \pi)^{-1 / 2} \int \mathrm{O}(\hbar \omega-\mathrm{E})\left[\mathrm{B} / \pi\left(\mathrm{E}^{2}+\mathrm{B}^{2}\right)\right] \mathrm{dE},
$$

where $\mathrm{O}(\hbar \omega)$ is given in Eqn. (10), by replacing $E_{n}$ by our previous results $E_{b}$, and the integration over $E$ is carried out from $-\infty$ to $+\infty$.

Figure ( $3 \mathrm{a}$ and $\mathrm{b}$ ) show the calculated total optical density, Eqn. (12), at three different parabolic quantum well width. The three curves in each figure have the same broadening parameter $\mathrm{B}=0.5$, and the same $\mathrm{Al}$ concentration $\mathrm{x}=0.15,0.25$ and 0.35 . 

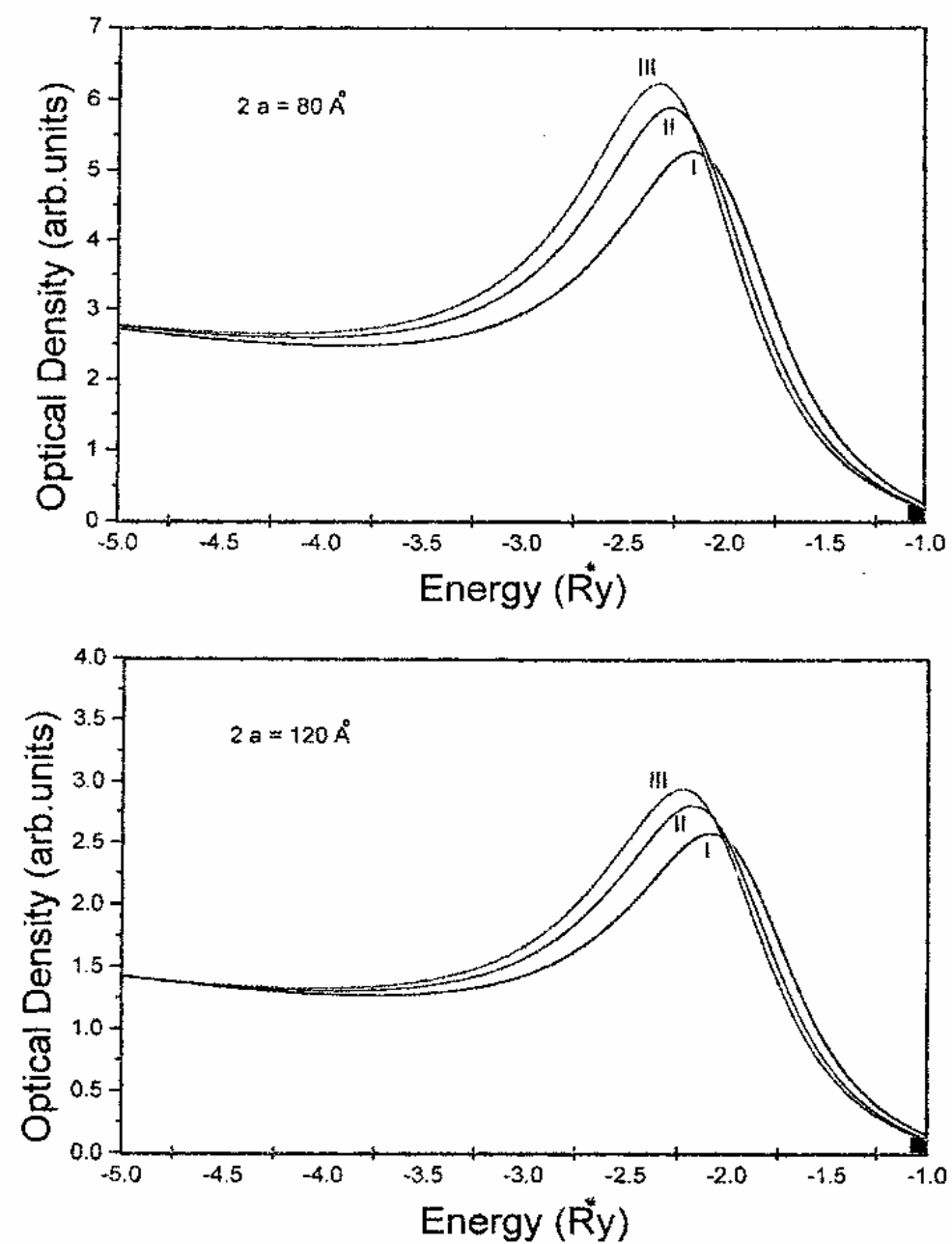

Fig. (3): The total optical density of the parabolic quantum wells Vs the photon energy in $\mathrm{R}_{\mathrm{y}}^{*}$. The three curves stand for: I) $\mathrm{x}=0.15$, II) $\mathrm{x}=0.25$ and III) $\mathrm{x}=0.35$.

Generally, in parabolic quantum wells we can tell that the total optical density increases by Al concentrations increasing. Also, the shift of the optical density peak is a noticeable one at small well width (Fig.3-a).

Again, as in the binding energy change Fig. (1-a), the shifts in the peak, almost, are the same at well width higher than $75 \AA$. A word in order can be said, from Fig. 3.a, we get the change in $\alpha(\Delta \alpha)$, equals to 0.1 , if we go from curve I to curve III, i.e., $\Delta \mathrm{x}=0.2$. But, in Fig. $3-\mathrm{c}, \Delta \alpha \approx 0.2$ with, $\Delta \mathrm{x}=0.2$, as we expected before (Fig.1-b). 
Consequently, at large values of $\alpha$, the total optical density will decrease, with almost equal shifts in the total optical density peak, at well width greater than 75A. Our results, qualitatively, are in a good coincidence with the previous results of the square well [17].

\section{Conclusions}

We have extended the concept of the fractional- dimensional spaces to the parabolic quantum well structures. This concept, allows quite good treatment for the optical density spectra due to Wannier- Mott excitons in parabolic wells. Since the finite parabolic quantum well has wide applications the present work is valuable for the understanding of the designing for the cases where the structure is used, especially, for the optical density spectrum in electro - optical modulators.

\section{Acknowledgment}

This work was submitted by one of us (H. M. H.) in partial fulfillment of the requirement of her Ph.D. degree.

\section{References}

1. M.Shayegan ,T.Sajoto, M.Santos and C.Silvestre, Appl. Phys. Lett., 53, 791 (1988), T.Sajoto, J.Jo, L.Engel, M.Santos and M.Shayegan, Phys. Rev., B39, 10464 (1989); M.Sundaram, A.C.Gossard, J.H.English, and M. Westervelt, Superlatt, Mictrostruct. 4, 683 (1988); E.G.Gwinn, M. Westervelt, P.F. Hopkins, A.J. Rimberg, M.Sundaram, and A.C.Gossard, Phys. Rev., B39, 6260 (1989); M.Sundaram, A.C.Gossard, P.O. Holtz, J.Appl. Phys., 69, 2370 (1991).

2. C.E. Hembree, B.A.Mason, J.T. Kwiatkowski , J.E. Furneaux, and J.A. linkman, Phys. Rev. B50, 15197(1994).

3. L.B. Liao, D. Heiman, P. F. Hopkins and A.C. Gossard, Phys. Rev., B49, 16825 (1994).

4. L. Wendler and T.Kraft, Phys. Rev., B54, 11436 (1996).

5. Walukiewicz, P.F.Hopkins, M. Sundaram, and A.C. Gossard, Phys. Rev. B44, 10909 (1991).

6. Pen Yuen, phys. Rev., B48, 17316 (1993).

7. Blinowski and T. Szwacka, Phys. Rev., B49, 10231 (1994).

8. X. Zang and M. L. Rustgi, Phys. Rev., B48, 2465 (1993) .

9. E. Hembree, B.A. Mason, A. Zhang, and J. A.Slinkman, Phys. Rev., 46, 7588 (1992).

10. M. P. Stopa and S. Das Sarma, Phys. Rev., B47, 2122 (1993).

11. Kang- Xian Guo and Shi-Wei Gu, Phys. Rev., B47, 16322 (1993) . 
12. Shao-hua Pan, Phys. Rev., B48, 2292 (1993).

13. F.H.Stillinger, J.Math. Phys. 18, 1224 (1977)

14. X..F.He, Solid State Commun. 61, 53 (1987).

15. H.Mathieu, P. Lefebvre, and P. Christor, Phys.Rev., B46, 4092 (1992).

16. Matos- Abiague, L.E. Oliveira, and M.de Dios-Leyva, Phys. Rev., B58, 4072 (1998).

17. P.Lefebvre, P.Christol, and H. Mathieu, Phys.Rev., B48, 7308 (1993).

18. G.Bastard, Phys.Rev., B24, 4714 (1981).

19. G.Oelgart, M.Proctor, D. Martin, F.Morier-Genaud, F.-K.Reinhart, B. Orschel, L.C.Andreani, and H.Rhan, Phys. Rev., B49, 10456 (1994).

20. V.Voliotis,R. Grousson, P. Lavallard, C. Gourdon and R. Planel, Phys. Rev., B52, 10725 (1995).

21. P.Dawson, K.J.Moore, G.Duggan,H.I. Ralph, and C.T.B.Foxon, Phys.Rev., B34, 6007 (1986).

22. H. Haug and S.W.Koch, Quantum Theory of Optical and Electronic Properties of Semiconductors (World Scientific, Singapore, 1990), pp. 148-176.

23. G. Bastard, Wave Mechanics Applied to Semiconductor Hetero- structures (Les Editions De Physique, Paris, 1988), pp. 237-295.

24. R. J. Elliott, Phys. Rev., 108, 1384 (1957).

25. X. F. He, Phys. Rev., B42, 11751 (1990). 